\title{
On selectivity and gating of ionic channels.
}

\author{
D. G. Luchinsky ${ }^{a, b}$, R. Tindjong ${ }^{a}$, P.V.E. McClintock ${ }^{a}$, \\ I. Kaufman ${ }^{c}$, R.S. Eisenberg ${ }^{d}$; \\ ${ }^{a}$ Department of Physics, Lancaster University, Lancaster LA1 4YB, UK; \\ ${ }^{b}$ Ames Research Center, MS 269-3, Moffett Field, CA, 94035, USA; \\ ${ }^{c}$ The Russian Research Institute for Metrological Service, \\ Gosstandart, Moscow, 119361, Russia; \\ ${ }^{d}$ Department of Molecular Biophysics and Physiology, Rush Medical College, \\ 1750 West Harrison, Chicago, IL 60612, USA;
}

\begin{abstract}
A novel conceptual model is introduced in which ion permeation is coupled to the protein wall vibration and the later in turn modulates exponentially strongly the permeation via radial oscillations of the potential of mean force. In the framework of this model of ion-wall-water interaction we discuss problems of selectivity between alike ions and coupling of ion permeation to gating.
\end{abstract}

Keywords: Ion channels, selectivity, gating, protein vibration

\section{INTRODUCTION}

Biological ion channels are natural conducting nanotubes in the membranes of cells and elementary building blocks of autonomous and central nervous system. E.g. half the metabolic energy consumed by the human brain is used by the ion pumps that move $\mathrm{K}^{+}$and $\mathrm{Na}^{+}$in and out of nerve cells. Understanding of the channel structure and operation will not only help to control and cure a vast range of diseases, but may also pave the way to building bio-electronics. Despite the impressive progress of international research on this subject in the past years, it remains the case that some fundamental features of the conduction mechanism are still eluding researchers. Indeed, over the last few years, the field of ion channel research has undergone spectacular development (see e.g. ${ }^{1}$ ). The molecular structures of a number of channels have been determined by crystallographic analysis, including the KcsA potassium channel ${ }^{2}$ that discriminates between $\mathrm{Na}^{+}$and $\mathrm{K}^{+}$. Furthermore, by detecting the size of structural fluctuations ${ }^{3}$ and conformational changes, ${ }^{4}$ it has become possible to provide the experimental information needed for molecular modelling of the dynamical features of the observed selectivity and gating. ${ }^{1,5}$

Substantial progress has also been achieved in modelling channel properties by use of molecular dynamics ${ }^{5}$ (MD), Brownian dynamics ${ }^{6}$ (BD), and Monte Carlo $^{7}$ (MC) simulations and by numerical solutions of the Poisson and Nernst-Planck ${ }^{8,9}$ (PNP) and reaction-rate (RR) equations. Note also that the MD is currently emerging as a reliable tool for calculating the potential of the mean force (PMF), including its radial dependence at the selectivity filter, that can be then used in the BD simulations. ${ }^{10,11}$ It is now well established that, in terms of decreasing accuracy and level of detail, existing approaches can be listed in order as: MD $\rightarrow \mathrm{BD} \rightarrow 3 \mathrm{D}-\mathrm{PNP} \rightarrow$ $1 D-P N P \rightarrow R R$. At each higher level, more and more degrees of freedom of the original many-body problem are being averaged out and, accordingly, less and less computer time is needed to estimate the channel properties in an increasingly approximate way an on increasingly coarse time scale.

Despite considerable progress, ion channel modelling can still be characterized as being at an initial stage: intuitive and "hand-waving" explanations of the permeation and selectivity of ions are only beginning to be replaced by quantitative statements based on rigorous physical laws. ${ }^{12}$ The main challenges faced in modelling

Further author information: (Send correspondence to R.T.)

R.T.: E-mail: r.tindjong@lancaster.ac.uk, 'Telephone: +44 (0)1524 593206, Address: Department of Physics, Lancaster University, Lancaster LA1 4YB, UK 
channel permeation include e.g very large number of degrees of freedom, long range interaction, widely-varying timescales, and fascinating diversity of properties and structures. No general methods exist at present that allow for derivation of ion channel properties from first principles on either time scale. Instead multiple adjustment procedures are used at every level (MD, BD, PNP, RR) to fit experimental results. Furthermore, averaging procedures cannot be rigorously justified at present (see e.g. ${ }^{13}$ ) and it seems very likely that degrees of freedom that provide a key to the understanding of conductivity, selectivity, and gating of the channel can inadvertently be lost in performing them. Even for MD simulations, many degrees of freedom have to be averaged out. Additional approximations are made in the transition from MD to BD simulations when the protein walls are assumed to be rigid, the water is treated as a continuous dielectric, and only thermal fluctuations are taken into account, whereas it is known ${ }^{1,5}$ that, in reality, wall dynamics, water structure, and charge fluctuations play important roles.

It was shown in our earlier work ${ }^{14,15}$ that vibrational degrees of freedom can be easily incorporated into the $\mathrm{BD}$ model of ion permeation and can lead to the strong modulation of the transition probabilities even in the case of water modelled as continuous dielectric and ions are treated as point charges. A number of the MC studies exists ${ }^{7,16}$ where the effect of volume exclusion was investigated considering ions as hard spheres and treating water as continuous dielectric. It is well known, however, that, ${ }^{17,}{ }^{18}$ in the bulk, the radial ion-ion distribution is strongly modulated by the structure of the water molecules. It is also well understood that at least in principle the ion permeation has to be coupled to the gating. However, there are only a few publications (see e.g. ${ }^{19,20}$ ) dealing with coupling of gating to ion permeation. The question of fundamental importance is, therefore, how the channel properties will be altered if the oversimplifying assumptions are lifted off.

In this paper we address this question directly by introducing a novel conceptual model that takes into account molecular structure of the water, size of the ions, and coupling of the ion's motion to the protein vibrations, largely neglected in the earlier research. We then discuss what alterations in the ion channels selectivity, conductivity, and gating can be expected within this model.

We report work in progress and begin by including a vibrational degree of freedom into the known BD model of channel permeation ${ }^{6,21}$ and focus on the boundary conditions and properties of ion-ion distribution in the bulk. Next a novel conceptual model that takes into account the molecular structure of water and the size of ions at the selectivity filter is formulated. An extension of this model that takes into account coupling of ion permeation to the gating dynamics is discussed. In conclusion we discuss the properties of the ion channel; conductivity, selectivity, and gating that can be simulated using this type of models.

\section{MODEL}

In this section we consider step-by-step essential effects that have to be included into the novel conceptual model to take into account molecular structure of the water, size of the ions, coupling of the ion's motion to the protein vibrations, charge fluctuations at the channel mouth, and amplification of the electrostatic interaction in the channel.

\subsection{Adding vibrational modes to the model of permeation}

Our first step is to extend the known ${ }^{6,21} \mathrm{BD}$ model of permeation based on the self-consistent solution of the coupled Poisson and equations of Brownian dynamics of ions. The model is extended by coupling Poisson and Langevin equations of ion dynamics to the equation describing the protein vibration at the selectivity filter. The resulting equations take the form

$$
\begin{gathered}
-\nabla \cdot(\varepsilon(\vec{r}) \nabla \phi(\vec{r}))=\rho(\vec{r}) \\
m_{i} \ddot{\vec{x}_{i}}=-m_{i} \gamma_{i} \dot{\vec{x}}_{i}+\sum_{j=1}^{N}\left[\frac{q_{i} q_{j}}{4 \pi \varepsilon \varepsilon_{0} r_{i j}^{2}}+\frac{9 U_{0} R_{c}^{9}}{r_{i j}^{10}}+A U_{0} \exp \left(\frac{R_{i j}-r_{i j}}{a_{e}}\right) \sin \left(2 \pi \frac{R_{i j}-r_{i j}}{a_{w}}-\alpha\right)\right] \frac{\vec{r}_{i j}}{r_{i j}} \\
+F_{c h}+\sqrt{2 m_{i} \gamma_{i} k_{B} T} \vec{\xi}_{i}(t)
\end{gathered}
$$




$$
\begin{aligned}
& m_{j} \ddot{\vec{x}}_{j}=-m_{j} \gamma_{j} \dot{\vec{x}}_{j}+\sum_{i=1}^{N}\left[\frac{q_{i} q_{j}}{4 \pi \varepsilon \varepsilon_{0} r_{j i}^{2}}\right.\left.+\frac{9 U_{0} R_{c}^{9}}{r_{j i}^{10}}+A U_{0} \exp \left(\frac{R_{i j}-r_{i j}}{a_{e}}\right) \sin \left(2 \pi \frac{R_{i j}-r_{i j}}{a_{w}}-\alpha\right)\right] \frac{\vec{r}_{j i}}{r_{j i}} \\
&+F_{c h}+\sqrt{2 m_{j} \gamma_{j} k_{B} T \vec{\xi}_{j}(t)} \\
& M \delta \ddot{R}=-M \Gamma \delta \dot{R}-K \delta R+\frac{Q q_{j}}{4 \pi \epsilon \epsilon_{0}\left((R+\delta R)^{2}+z^{2}\right)}+\sqrt{2 M \Gamma k_{B} T} \nu(t)
\end{aligned}
$$

Here $\vec{r}_{i j}=\vec{x}_{i}-\vec{x}_{j}, r_{i j}=\left|\vec{r}_{i j}\right|, A=\sqrt{1+\left(\frac{a_{w}}{2 \pi a_{e}}\right)^{2}}, \alpha=\arctan \left(\frac{a_{w}}{2 \pi a_{e}}\right)$. Where $m_{i}, \vec{x}_{i}$ and $q_{i}$ are the mass, position and charge of the $i$-th ion. In the Brownian dynamics simulations, water molecules are not taken into account explicitly and are represented by an average frictional force with a friction coefficient $m_{i} \gamma_{i}$ and a stochastic force $\sqrt{2 m_{i} \gamma_{i} k_{B} T} \vec{\xi}_{i}(t)$ arising from random collisions. The long range Coulomb interaction is represented by the $1 / r$ potential. The addition of the pairwise repulsive $1 / r^{9}$ soft-core interaction potential insures that ions of opposite charge, attracted by the inter-ion Coulomb force, do not collide and neutralize each other. $\vec{r}_{i j}$ is the distance between ions $i$ and $j . U_{0}$ and $R_{c}$ are respectively the overall strength of the potential and the contact distance between ions pairs. The oscillating part is added to the potential and takes explicitly into account the internuclear separation for the two solvents, where $a_{w}$ is the oscillation length, $a_{e}$ the exponential drop parameter and $R_{i j}$ is the origin of the hydration force which is slightly shifted from $R_{c}$ by +0.2 Angstrom for like ions and -0.2 otherwise. ${ }^{22} F_{c h}$ is the dielectric force in the channel obtained by solving Poisson equation numerically using finite volume methods (FVM). ${ }^{23}$ We use the Langevin equation to model the collective motion of the atoms forming the channel protein charged ring located at the selectivity filter. In this way, our analysis is based on the assumption that the movement of structural domains of the channel protein may be described as the motions of independent, elastically bound Brownian particles. ${ }^{24}$ We have included the damping term $M \Gamma \delta \dot{R}$ and the corresponding random force $\sqrt{2 M \Gamma k_{B} T} \nu(t)$, whose amplitude is related to the damping constant via the fluctuation-dissipation theorem. The function $\nu(t)$ is a Gaussian white noise. $Q$ is the total fixed charge on the flexible ring interacting with an ion of charge $q_{j}$ on the channel axis $z . R$ is the channel radius, $\delta R$ is a small variation of the channel radius and $K$ is the elastic constant of the channel's protein. In the particular case of the Gramicidin A (GA) channel, the value of the elastic constant is estimated by calculating the root mean square deviation (RMSD) of the backbone forming its central part, together with the single ion potential of a $\mathrm{K}^{+}$ion as it traverses the channel. Using the data from, ${ }^{25}$ we obtain an elastic constant of $\sim 1.6577 \mathrm{~N} / \mathrm{m}$ for a maximum RMSD of $1 \AA$. The GA channel molecular weight $M$ is about $4 k D a=6.64 \times 10^{-24} \mathrm{~kg}^{26}$ The diffusion constant of the protein in the membrane is between $10^{-14}$ to $10^{-16} \mathrm{~m}^{2} / \mathrm{s}^{27}$

Note that this model includes the effect of hydration on the ion-ion distribution in the bulk, which turns out to be crucial for an understanding of the effect of hydration at the selectivity filter. It also allows us to analyze other important properties of the permeation mechanism including e.g. charge fluctuations at the channel mouth, amplification of the electrostatic interaction in the channel, and the modulation of the axial potential of the mean force by the moving wall. We now consider briefly these effects.

\subsection{Effect of hydration}

As we have mentioned in the introduction one of the most strong assumptions made in modelling channel permeation is that water in the channel is considered as continuous dielectric. This assumption is not very realistic because the channel diameter at the selectivity filter is of the order of $10 \AA$. At the same time it is well known ${ }^{17,18}$ that, in the bulk, the radial ion-ion distribution is strongly modulated by the structure of the water molecules. As a result the distribution has a few sharp peaks on a spatial scale of $\sim 12 \AA$ with separations proportional to the ionic diameter.

To see how the hydration effect can be taken into account at the selectivity filter we consider first the effect of hydration in the bulk following the simplified description adopted in. ${ }^{6,21}$ The parameters of the ion-ion interaction used in the calculations are presented in Table. 1. 


\begin{tabular}{|l|r|r|r||r|l|}
\hline Ions & $U_{0}\left[\mathrm{k}_{B} \mathrm{~T}\right]$ & $R_{c}[\AA]$ & $R[\AA]$ & $a_{w}[\AA]$ & $a_{e}[\AA]$ \\
\hline $\mathrm{Na}-\mathrm{Na}$ & 0.5 & 3.50 & 3.7 & & \\
$\mathrm{Na}-\mathrm{Cl}$ & 8.5 & 2.76 & 2.53 & 2.76 & 1.4 \\
$\mathrm{Cl}-\mathrm{Cl}$ & 1.4 & 5.22 & 5.42 & & \\
\hline
\end{tabular}

Table 1: Parameters used in the calculation of the short range ion-ion interaction with hydration

The following parameters are used for the simulations:

Dielectric constants: $\varepsilon_{2}=80, \varepsilon_{1}=2$;

Masses (in kg): $m_{N a}=3.8 \times 10^{-26}, m_{C l}=5.9 \times 10^{-26}$;

Diffusion coefficients (in $\mathrm{m}^{2} \mathrm{~s}^{-1}$ ): $D_{N a}=1.33 \times 10^{-9}, D_{C l}=2.03 \times 10^{-9}$,

(Note that $\mathrm{D}$ is related to the friction coefficient via $D=\frac{k_{B} T}{m \gamma}$ );

Ion radii (in $\AA$ ): $r_{N a}=0.95, r_{C l}=1.81$;

Temperature: $T=298 \mathrm{~K}$.

In the absence of the hydration forces the radial distribution of the ions are smooth as shown in the Fig. 1(left). The overlap of the electron clouds induces a strong modulation of the radial probability distribution of ions as can be observed in the Fig. 1(right). Because the channels are filled with water molecules it is natural to assume that the effect of dipole of the water molecules will play an important role in the radial ion distribution at the selectivity site. The shape of this distribution will depend strongly on the size of the ions and on the parameters
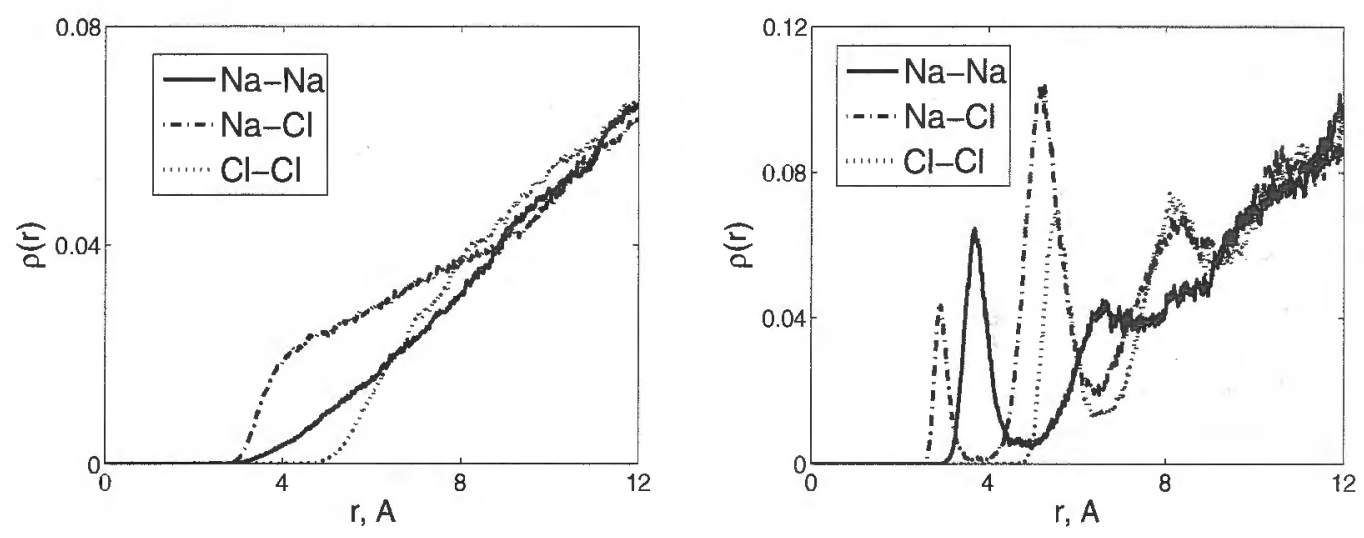

Figure 1. (left)number of ions as a function of the distance between ions of different type: $\mathrm{C}=400 \mathrm{mMol}$; Box size: 40 A. Forces included into the simulations: (i) Coulomb interaction; (ii) short range repulsion. (Right) The number of ions as a function of the distance between ins of different type: $\mathrm{C}=400 \mathrm{mMol}$; Box size: 40 A. Forces included into the simulations: (i) Coulomb interaction; (ii) short range repulsion; (iii) hydration.

of the ion-wall interaction that are specific for each ion species. The effect of the dielectric self-energy in the channel will further modulate this distribution in such a way that the probability to find ion near the channel wall will be exponentially small. It is clear that taking into account the molecular structure of the water and the effect of overlapping of the electronic clouds will result in a complex spatially distributed potential profile near the selectivity filter with high ion specific potential barriers. Therefore, at many physiological conditions, this factor may play and important, even a dominating role in the selectivity of the ionic channels. It is also clear that fluctuations of the position of the protein at the selectivity site will induce a strong modulation of the height of the potential barrier enhancing probabilities of the transition of the ions through the channel. 


\subsection{Effect of moving wall}

We now turn to the discussion of the effect of the wall vibrations on the protein permeation. The system considered (see Fig. 2) is made of three compartments of equal size. The middle block constitutes the protein through which there is a cylindrical hole approximating the open channel. To model ion coupling to the vibration modes of the channel we introduce a moving segment of the protein wall elastically bind to the wall. The moving segment is charged and represents the selectivity site. Dynamics of the moving segment and of the ions in the bath and channel are modeled using Brownian Dynamics (BD) simulations, see eqs. (2)-(4). At this stage we still keep the assumption that the water in the channel is a continuous dielectric and consider the modulation of the axial potential faced by ions in the channel by the moving selectivity filter. The corresponding in the axial
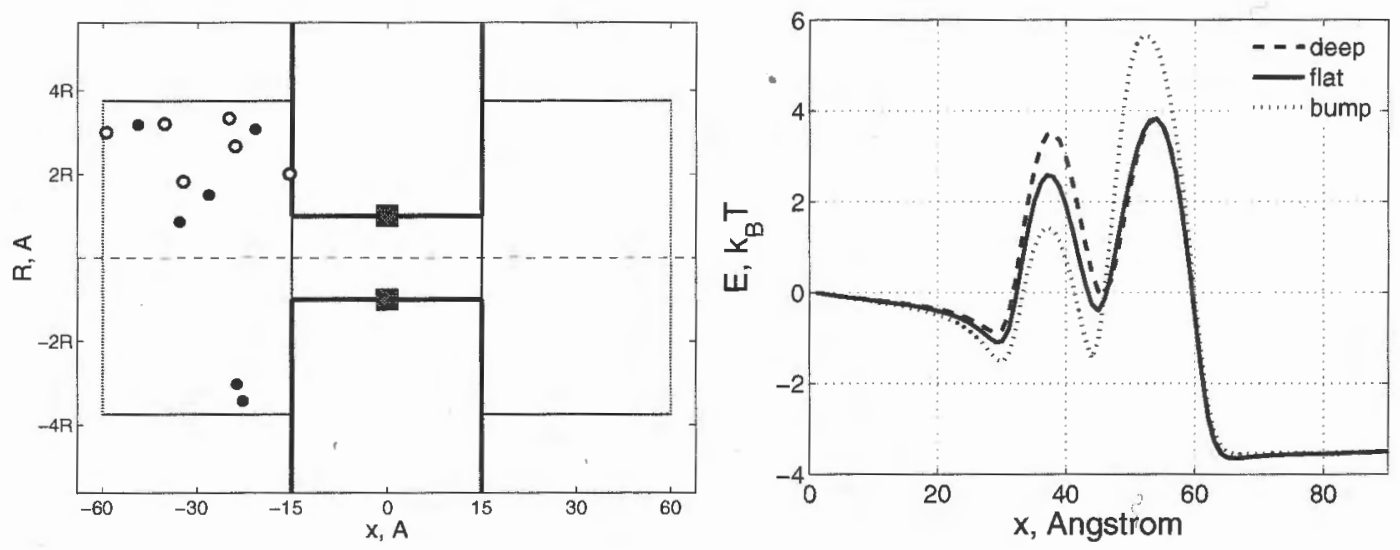

Figure 2. (left) Sketch of the model with the middle block representing the protein through which there is a cylindrical hole approximating the open channel. To moving charged segment of the protein wall elastically bind to the wall is shown by the filled squares. Negative ions are shown by the blue circles, positive ions are shown by the red circles. (Right) axial potential faced by a $\mathrm{Na}^{+}$ion for channel with regular contour (flat), channel with a rectangular bump at the middle (bump, moved 1 Åtowards the channel axis) and a channel with a rectangular hole at its middle (deep, moved $1 \AA$ away from the channel axis).

potential for three different positions of the selectivity filter areshown in the Fig. 2(Right). A very interesting phenomenon of pumping ions in and out of the channel can be noticed from this figure. Indeed, when the selectivity filter moves deeper inside the channel (dotted line) the potential barrier for the ion to enter the channel is lowered. When, on the other hand, the selectivity filter moves away from the channel axis (dashed line) the potential barrier for the ion to exit the channel is lowered, while the potential barrier for the ion to return to the original location becomes higher.

It is clear from the present consideration that for this effect to have considerable influence on the ion permeation the frequencies of the protein wall vibration must be of the order of the inverse time of the ion permeation (i.e. of the order of $10^{7}-10^{8} \mathrm{~Hz}$ ). Indeed, for the effect to be most pronounced the selectivity filter should be in its lowest position at the time ion enters the channel and in its highest position at the time ion exits the channel. The frequency of the wall vibration is taken to be the same as the one of the local motion of proteins. It is known from the literature that this correspond to atomic fluctuation whose frequencies are of the order of $10^{12}-10^{15} \mathrm{~Hz}$ and with amplitudes less than $1 \AA$. We will show later in this paper that the protein vibration can have an exponentially strong effect both on the transition rates and on the selectivity of the ion channels when the structure of the water molecules at the selectivity filter is taken into account.

\subsection{Amplification of the electrostatic interaction in the channel}

To build a realistic low-dimension BD model of ion permeation it is also very important to take into account the effect of the channel walls on the strength of the electrostatic interaction between the ions in the aqueous 

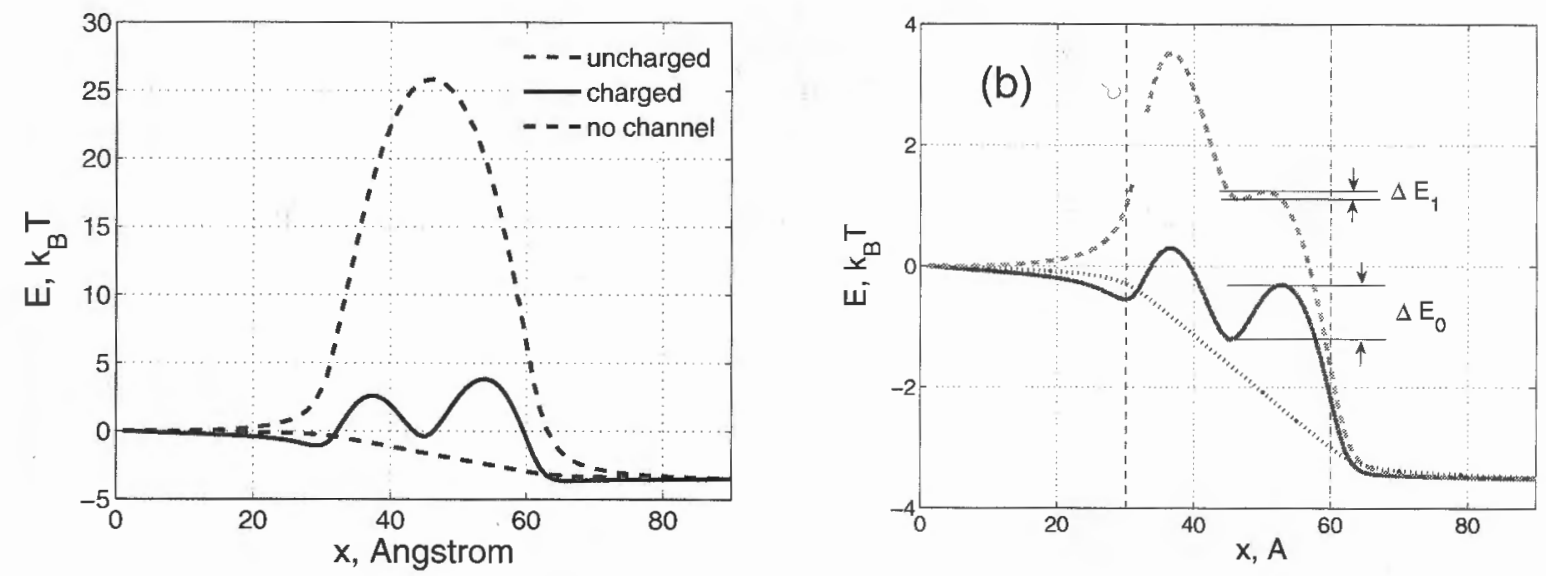

Figure 3. (a) The potential energy profiles in a cylindrical channel of radius $\mathrm{r}=4 \AA$ when an electric field of $10^{7} \mathrm{~V} / \mathrm{m}$ is applied in the $\mathrm{z}$ direction. The solid (blue) and dashed (red) lines correspond to the channels with and without fixed charges, respectively. The profile of a passive channel $\left(\epsilon_{\text {protein }}=80\right)$ is indicated by the dotted (black) line. The channel is situated between 30 and $60 \AA$. (b) Total energy of the channel as a function of the position of the ion when: the first ion is located at the channel mouth on the left ] (dashed line); the channel is empty (solid line); no channel (dotted line). Vertical dashed-dotted lines show channel entrance. The height of the potential barrier for the ions at the selectivity site and no ion at the channel mouth is denoted $\Delta E_{0}$. In the presence of the second ion in the channel mouth this barrier is reduced to $\Delta E_{1}$.

solution. We will show in this section that ion channels can be viewed as an amplifiers of such an electrostatic interaction. To see this we, first, analyze the energy profile faced by one ion moving through the channel.

The total electrostatic energy required to move one ion from the bulk solution to a bare channel that is 30 Ålong and 4 Åin radius as a function of the position of the ion is calculated as shown in the Fig. 3(a). The potential drop across the channel is $\Delta \Phi=90 \mathrm{mV}$, the charge at the selectivity site (at the middle of the channel) is -1e. Note that the value of the potential barrier for the ion at the selectivity site to exit the channel is $\Delta E_{0}$ as shown in the Fig. 3 (b).

We now consider the reduction of this potential barrier induced by the second ion situated at the channel mouth. The total energy of the channel as a function of the position of the first ion moving along the channel when the second ion is located at the channel mouth is shown in the Fig. 3(b) by dashed line. It can be seen that the presence of a second ion at the channel entrance decreases the energy barrier to $\Delta E_{1}$. We note the reduction of the potential barrier from $\Delta E_{0}$ to $\Delta E_{1}$ is much stronger in the channel with low dielectric constant. I.e. the effect of charge fluctuation is strongly enhanced by the protein low dielectric constant compared to water. In the absence of the protein walls the interaction between two ions will be purely due to the Coulomb forces and the corresponding reduction of the potential barrier $\Delta E_{C}=\frac{e^{2}}{4 \pi \epsilon_{2} \epsilon_{0} r_{12}}$ will be much smaller then the effect induced by the channel $\Delta E_{0}-\Delta E_{1}$.

I.e. channel can be viewed as an amplifier of the electrostatic interaction in aqueous solutions. This effect can be readily understood by noticing that the Coulomb interaction between two changes in the dielectric media is inversely proportional to the dielectric constant of the media. I.e. for the same distance between the ions the interaction in the protein $\left(\epsilon_{\text {protein }}=2\right)$ is 40 times stronger then in water $\left(\epsilon_{\text {water }}=80\right)$. Clearly in the protein channel filled with water the strength of the interaction is somewhere in between of these two extreme values. And the smaller radius of the channel the closer the strength of the interaction to its upper value in protein.

\subsection{Effect of charge fluctuations}

As we have shown in the previous section due to the effect of amplification the modulation of the potential barrier by the charge at the channel mouth is much stronger then the effect of thermal fluctuations. Therefore, the fluctuation of the charge at the channel mouth, which were largely neglected in the earlier work, may have 
a dominating (as compared to the thermal fluctuations) contribution to the transition probabilities. To take this effect into account we have estimated the distributions of the charge fluctuations from the simulations by recording continuously (during a few microseconds) the total positive and negative charge in the channel mouth with volume $v_{M}=\pi r^{3}$, where $r=6 \AA$. The arrival time was estimated by recording the interval of times between the subsequent events of ion arrival to the channel mouth. The results of the simulations are summarized in Fig. 4. It can be seen from the figure that the arrival time distribution follows an exponential distribution with mean arrival time $\tau_{a r} \sim 3.6 \times 10^{-9} \sec$ for $\mathrm{Na}^{+}$and $\tau_{a r} \sim 4.7 \times 10^{-9} \mathrm{sec}$ for $\mathrm{Cl}^{-}$.
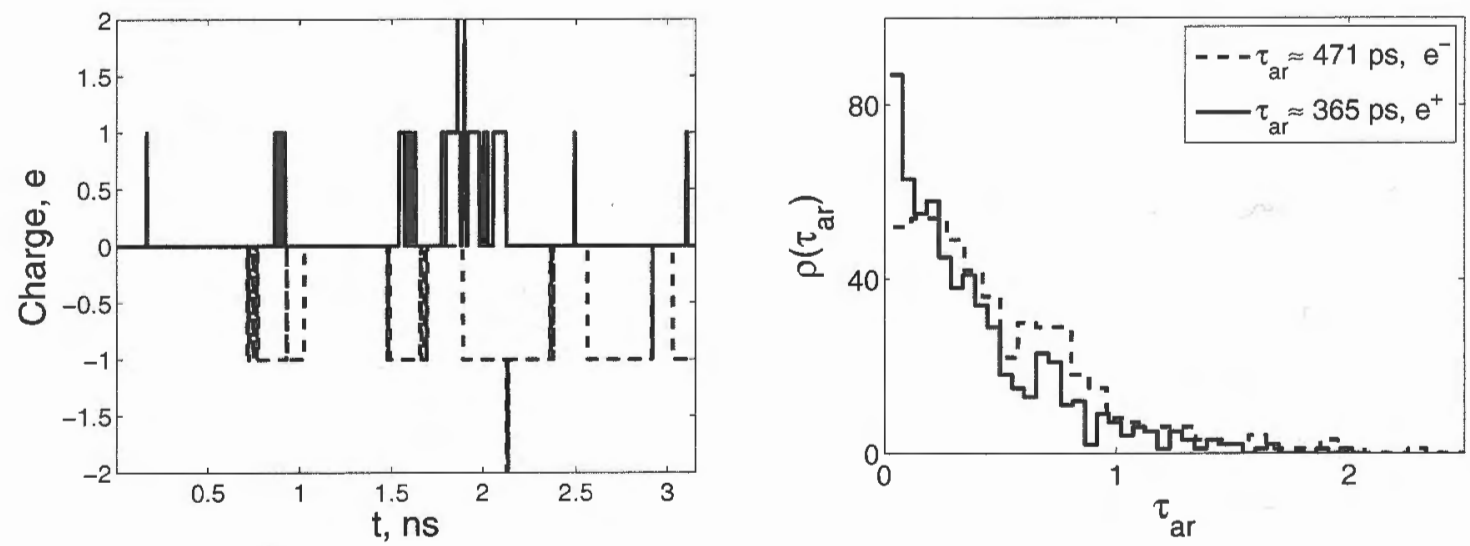

Figure 4. (Left) Fluctuations of the charge at the mouth entrance. (Right) The arrival time distributions for positive (solid line) and negative (dashed line) ions for cylindrical channel of radius $\mathrm{r}=6 \AA$.

\subsection{Adding effects of hydration and charge fluctuations at the selectivity filter}

In the previous sections we have described a number of phenomena that have a leading order contribution to the selectivity and gating of ion channels including protein vibration and hydration effects at the selectivity filter and effect of the amplification of the charge fluctuations at the channel mouth. We now introduce a lowest order BD model of channel permeation that takes these effects into account. To do so we consider a singly occupied channel, notice that all the forces acting on the ion and the selectivity filter are additive, and write Langevin equations describing ion's axial and selectivity filter radial dynamics in the form

$$
\begin{aligned}
& -\nabla \cdot(\varepsilon(\vec{r}) \nabla \phi(\vec{r}))=\rho(\vec{r}) \\
& m_{j} \ddot{\vec{x}}_{j}=-m_{j} \gamma_{j} \dot{\vec{x}}_{j}+F_{H}\left(x_{j}, R\right)+F_{V}\left(x_{j}, R\right)+F_{c h}\left(x_{j}\right)+\sum_{i=0}^{N} F_{A m}\left(x_{j}, x_{i}\right)+\sqrt{2 m_{j} \gamma_{j} k_{B} T} \vec{\xi}_{j}(t) \\
& M \delta \ddot{R}=-M \Gamma \delta \dot{R}-K \delta R+F_{H}\left(R, x_{j}\right)+F_{V}\left(R, x_{j}\right)+\sqrt{2 M \Gamma k_{B} T} \nu(t)
\end{aligned}
$$

Here the first equation describes coupling of the ion and protein dynamics to the electrostatic potential in the channel and $\vec{r}=\left\{x_{j}, R, x_{1}, \ldots, x_{N}\right\}$, where $R$ is the location of the moving selectivity filter, $x_{1}, \ldots, x_{N}$ are axial coordinates of $\mathrm{N}$ ions at the channel mouth at the left and right entrances (note that to a good approximation $N \leq 4$ ). The position of the ion in the channel its mass and charge are $x_{j}, m_{j}$, and $q_{j}$ respectively.

The channel force $F_{c h}\left(x_{j}\right)$ is the same as in the original model (1)-(4). It corresponds to the energy profile faced by one ion moving through the channel and is shown e.g. in the Fig. 3(left) by the solid line.

Coupling to the protein vibration. The term that describes coupling of the ion permeation to the protein vibration is taken in the simplest form of the Coulomb interaction between the ion and the charge at the selectivity filter

$$
F_{V}\left(x_{j}, R\right)=\frac{Q q_{j}}{4 \pi \epsilon \epsilon_{0}\left((R+\delta R)^{2}+z^{2}\right)}
$$


$F_{V}\left(x_{j}, R\right)$ and $F_{V}\left(R, x_{j}\right)$ differ by the sign. Note that the corrections to this simple Coulomb interaction due to the induced charge in the protein dielectric are included into the $F_{c} h$ term for the ion axial motion, but are neglected for the protein motion.

Hydration. One of the main results of the present research is modeling of the hydration effect at the selectivity site and an understanding of how this effect can modify exponentially the selectivity and permeation of ion, once the ions motion is coupled to the protein vibration. As a first order of approximation one can try to model these forces using results obtained for the hydration forces of ion-ion interaction in the bulk ${ }^{17}$ and applying further simplification alone the lines suggested in. ${ }^{6,21}$ To this end we introduce the model of hydration forces at teh selectivity filter in the form

$$
F_{H}\left(x_{j}, R\right)=\left[\frac{9 U_{W 0} R_{W c}^{9}}{r^{10}}+A_{W} U_{W 0} \exp \left(\frac{R_{W}-r}{a_{W e}}\right) \sin \left(2 \pi \frac{R_{W}-r}{a_{W w}}-\alpha_{W}\right)\right] \frac{\vec{r}}{r},
$$

where parameters have the same meaning as in the bulk (see Sec. 2.1 but may have different values. In particular, we denote all the parameters of hydration forces at the selectivity filter with an additional subscript $W$ and assume that $r$ is the distance between ion and the selectivity filter, $A_{W}=\sqrt{1+\left(\frac{a_{W w}}{2 \pi a_{W e}}\right)^{2}}$ and $\alpha_{W}=$ $\arctan \left(\frac{a_{W w}}{2 \pi a_{W e}}\right)$. As before $U_{W 0}$ and $R_{W c}$ are respectively the overall strength of the potential and the contact distance between ions pairs, $a_{W} w$ is the oscillation length, $a_{W e}$ is the exponential drop parameter, and $R_{W}$ is the origin of the hydration force which is slightly shifted from $R_{W^{\prime}}$. At this stage, however, all these parameters become fitting experimental parameters, whose values are to be compared with the first principle calculations based e.g on the methods described in ${ }^{10,11}$ if the assumption of the cylindrical symmetry will be lifted off and more realistic models of the water molecules (see e.g. ${ }^{17}$ ) will be adopted. As above $F_{H}\left(x_{j}, R\right)$ and $F_{H}\left(R, x_{j}\right)$ differ by the sign. Note also that in principle an additional important common multiplier has to be used for the hydration forces to reflect the radial dependence of this force due to the presence of the dielectric channel walls. However, in our model the ions are restricted to move alone the channel axis, where the contribution due to the dielectric channel walls can be neglected.

Charge fluctuations. Another important contribution to the channel permeation, and more specifically coupling of the permeation to the bathing compositions, is described by the term $\sum_{i=0}^{N} F_{A m}\left(x_{j}, x_{i}\right)$. It corresponds to the large changes in the energy profile faced by an ion in the channel induced by the presence of other ions at the channel mouth. Note that this term is taken into account implicitly in the earlier BD simulations. ${ }^{6}$ The results of the present research, described briefly in Secs. 2.4 and 2.5 and in more details in, ${ }^{28}$ allow one to include this effect in the analytical $R R$ calculations. Indeed, taking into account the fact that the charge fluctuations at the channel mouth occur at the sub-nanosecond time scale with exponential distribution of the arrival times, one can approximate this term (as will be described in more details elsewhere) by a Poisson random force with some experimentally determined distribution of the modulation amplitudes. In this way both the BD simulations of the channel permeation and selectivity for the complex bath compositions can be substantially simplified. And most importantly, the corresponding RR calculations can be substantially improved since the charge fluctuations induced contribution into the transition rate at the selectivity site can dominate over the contribution induced by thermal fluctuations under many physiologically relevant conditions.

\subsection{Coupling ion permeation to the gating}

Explanation of the mechanisms of channel gating in response to an applied voltage (see e.g., ${ }^{29}$ external and internal ligand binding, ${ }^{30}$ stresses $^{31}$ and other perturbations is one of the most challenging problem in channel research. The main focus so far, however, has been on RR theories and MD modelling, with only a few publications ${ }^{19,20}$ dealing with coupling of gating to the ion motion or calculations of the potential barriers imposed on the ions by gating. At the same time, the energy of the ion-wall interaction is comparable with the energy barrier separating closed and open states of the ion channels. ${ }^{32}$ Furthermore, in their open conducting state, channels still fluctuate between short-lived closed and open states, which has apparently no physiological significance. These fluctuations often occur as bursts. It is of particular interest to investigate if and how the protein vibration affects the energetics of gating, how the potential profiles experienced by ions during 
permeation are built in the various conformational states, and how known features of the dwell-time distribution can be incorporated into this physical picture.

The conceptual model (5) allows us to include the coupling of the ion permeation to the gating in the form of bistable potential for the wall vibrations. Importantly, this model suggest how to calculate the shape of the gating potential experienced by an ion at the selectivity site from the first principles using MD simulations. Furthermore, it becomes possible to relate the sensitivity of gating to external conditions by considering a combination of two (or more) double-well potentials: one with a deep long-lived closed state, and one with two almost equally deep short-lived open and closed states. The transition rate between the two shapes of potential is determined in each case by the external conditions.

\subsection{Coupling ion permeation to the gating}

Explanation of the mechanisms of channel gating in response to an applied voltage (see e.g., ${ }^{29}$ external and internal ligand binding, ${ }^{30}$ stresses $^{31}$ and other perturbations is one of the most challenging problem in channel research. The main focus so far, however, has been on RR theories and MD modelling, with only a few publications ${ }^{19,20}$ dealing with coupling of gating to the ion motion or calculations of the potential barriers imposed on the ions by gating. At the same time, the energy of the ion-wall interaction is comparable with the energy barrier separating closed and open states of the ion channels. ${ }^{32}$ Furthermore, in their open conducting state, channels still fluctuate between short-lived closed and open states, which has apparently no physiological significance. These fluctuations often occur as bursts. It is of particular interest to investigate if and how the protein vibration affects the energetics of gating, how the potential profiles experienced by ions during permeation are built in the various conformational states, and how known features of the dwell-time distribution can be incorporated into this physical picture.

The conceptual model (5) allows us to include the coupling of the ion permeation to the gating in the form of bistable potential for the wall vibrations. Importantly, this model suggest how to calculate the shape of the gating potential experienced by an ion at the selectivity site from the first principles using MD simulations. Furthermore, it becomes possible to relate the sensitivity of gating to external conditions by considering a combination of two (or more) double-well potentials: one with a deep long-lived closed state, and one with two almost equally deep short-lived open and closed states. The transition rate between the two shapes of potential is determined in each case by the external conditions.

\section{SUMMARY AND CONCLUSIONS}

In this work we consider the following problem: How molecular structure of the water and coupling of the ion's motion to the protein vibrations can affect selectivity and permeation of the ionic channels. We begin by revising $\mathrm{BD}$ model of the ion-ion interaction in the bulk which shows that the ion-ion distribution in the bulk at the distance between ions of the order of $10 \AA$ is dominated by the hydration effect. It is important to note that these results are confirmed by the experimental observations. We then proceed by showing that the protein vibration at the selectivity filter can modify substantially the axial potential for the ion and lead to the effect of pumping ions in and out of the channel. Next we consider the effect of the amplification of the charge fluctuations at the channel mouth by the channel walls and show that the effect of charge fluctuations can dominate the rate of ion transition through the channel as compared to the effect of thermal fluctuations. Finally, we introduce a conceptual model that takes into account both the effect of hydration at the selectivity filter and the coupling of permeation to the protein wall vibrations. We also show how the effect of the charge fluctuations can be incorporated into this model and discuss briefly the coupling of ion permeation to the gating mechanism within this model.

One of the central results is the modelling of the hydration at the selectivity filter and its coupling to the protein vibration. Within this model it becomes possible to explain in a self-consistent way the strong selectivity of the channel between alike ions (such as e.g. selectivity between $\mathrm{Na}^{+}$and $\mathrm{K}^{+}$in the $\mathrm{Kcs}$ A channel) that coexists with very high transition rates of ions through the channel. To see this we note (see Fig. 1(Right)) that the ion-ion distribution for alike ions is sharply peaked at periodic locations determined mainly by the ion diameter. Furthermore, the law dielectric constant of the channel walls will force ions to occupy predominantly the narrow 
region along the channel axis. As a result it will be the part of the hydration potential situated approximately at one channel radius distance from the selectivity filter wall that affect mostly the ions permeation. It can be seen from the Fig. 1(Right) that exactly at this distances the hydration potential can discriminate 1000:1 (and more) between different species (notice the difference in the probability to find $\mathrm{Na}^{+}$next to $\mathrm{Na}^{+}$at this distance as compared to the corresponding probability for $\mathrm{Cl}-\mathrm{Cl}$ distribution). In practice, this will correspond to the deeper potential well for the preferable species at the selectivity site, i.e. the channel will pump in mainly this species of ions. However, a small change (of the order of 1 Aor less) in the radial location of the selectivity filter may result in the dramatic changes in the distributions. And, in particular, the potential well for $\mathrm{Na}^{+}$ ions will change to a potential barrier, thus pumping $\mathrm{Na}^{+}$ions out of the channel. I.e. we see that coupling of the hydration potential at the selectivity filter to the protein vibration can amplify exponentially strongly the pumping effect described in the Sec. 2.3.

It is interesting to note that within the same conceptual model other important effects such as stress relaxation, energy dissipation, and coupling of the ion permeation to the gating mechanism can also be addressed in a self-consistent way. The corresponding work is currently underway and will be reported elsewhere.

\section{ACKNOWLEDGMENTS}

The work was supported by the Engineering and Physical Sciences Research Council (UK), RFBR (The Russian Foundation for Basic Research), INTAS, and ESF.

\section{REFERENCES}

1. B. Corry, "Understanding ion channel selectivity and gating and their role in cellular signalling," Molecular Biosystems 2(11), pp. 527-535, 2006.

2. D. A. Doyle, J. Cabral, atilde, M. o, R. A. Pfuetzner, A. Kuo, J. M. Gulbis, S. L. Cohen, B. T. Chait, and R. MacKinnon, "The structure of the potassium channel: Molecular basis of $\mathrm{k}+$ conduction and selectivity," Science 280(5360), pp. 69-77, 1998.

3. Y. Zhou, J. H. Morais-Cabral, A. Kaufman, and R. MacKinnon, "Chemistry of ion coordination and hydration revealed by a $\mathrm{K}^{+}$channel-Fab complex at 2.0 Angstrom resolution," Nature 414, pp. 43-48, 2001.

4. R. Dutzler, E. B. Campbell, M. Cadene, B. T. Chait, and R. MacKinnon, "X-ray structure of a cic chloride channel at 3.0 angstrom reveals the molecular basis of anion selectivity," Nature 415(6869), pp. 287-294, 2002 .

5. S. Y. Noskov, S. Berneche, and B. Roux, "Control of ion selectivity in potassium channels by electrostatic and dynamic properties of carbonyl ligands," Nature 431(7010), pp. 830-834, 2004.

6. S.-H. Chung, T. W. Allen, and S. Kuyucak, "Modelling divers range of potassium channels with Brownian dynamics," Biophys. J. 83, pp. 263-277, 2002.

7. D. Boda, D. Gillespie, W. Nonner, D. Henderson, D. Busath, and B. Eisenberg, "Effects of dielectrics on selectivity: Computing induced charge in monte carlo simulations," Biophysical Journal 86(1), pp. 6A-6A, 2004. Part 2 Suppl. S.

8. A. B. Mamonov, R. D. Coalson, A. Nitzan, and M. G. Kurnikova, "The role of the dielectric barrier in narrow biological channels: A novel composite approach to modeling single-channel currents," Biophys. $J$. 84(6), pp. 3646-3661, 2003.

9. B. Dezso, V. Monika, E. Bob, N. Wolfgang, H. Douglas, and G. Dirk, "The effect of protein dielectric coefficient on the ionic selectivity of a calcium channel," The Journal of Chemical Physics 125(3), p. 034901, 2006.

10. T. Bastug, A. Gray-Weale, S. M. Patra, and S. Kuyucak, "Role of protein flexibility in ion permeation: A case study in gramicidin a," Biophysical Journal 90(7), pp. 2285-2296, 2006.

11. T. W. Allen, O. S. Andersen, and B. Roux, "Ion permeation through a narrow channel: Using gramicidin to ascertain all-atom molecular dynamics potential of mean force methodology and biomolecular force fields," Biophysical Journal 90(10), pp. 3447-3468, 2006. 
12. S. H. Chung and B. Corry, "Three computational methods for studying permeation, selectivity and dynamics in biological ion channels," Soft Matter.1(6), pp. 417-427, 2005.

13. B. Nadler, T. Naeh, and Z. Schuss, "Connecting a discrete ionic simulation to a continuum," Siam Journal on Applied Mathematics 63(3), pp. 850-873, 2003.

14. R. Tindjong, A. Applegate, R. S. Eisenberg, I. Kaufman, D. G. Luchinsky, and P. V. E. McClintock, "Ionic current through an open channel: a low-dimensional model of coupling with vibrations of the wall," in Fluctuations and Noise in Biological, Biophysical, and Biomedical Systems Ii, D. Abbott, S. M. Bezrukov, A. Der, and A. Sanchez, eds., Proceedings of the Society of Photo-Optical Instrumentation Engineers (Spie) 5467, pp. 338-344, 2004. Conference on Fluctuations and Noise in Biological, Biophysical and Biomedical Systems II MAY 26-28, 2004 Maspalomas, SPAIN.

15. R. Tindjong, R. S. Eisenberg, I. Kaufman, D. G. Luchinsky, and P. V. E. McClintock, "Brownian dynamics simulations of ionic current through an open channel," in Noise and Fluctuations, T. Gonzalez, J. Mateos, and D. Pardo, eds., Aip Conference Proceedings 780, pp. 563-566, 2005. 18th International Conference on Noise and Fluctuations SEP 19-23, 2005 Salamanca, SPAIN.

16. D. Boda, D. D. Busath, B. Eisenberg, D. Henderson, and W. Nonner, "Monte carlo simulations of ion selectivity in a biological na channel: Charge-space competition," Physical Chemistry Chemical Physics 4(20), pp. 5154-5160, 2002.

17. L. X. Dang and B. M. Pettitt, "Solvated chloride ions at contact," The Journal of Chemical Physics 86(11), pp. $6560-6561,1987$.

18. X. D. Liem, E. R. Julia, and A. K. Peter, "The effect of water models on the interaction of the sodiumchloride ion pair in water: Molecular dynamics simulations," The Journal of Chemical Physics 93(10), pp. $7528-7529,1990$.

19. K. Lee and W. Sung, "Ion transport and channel transition in biomembranes," Physica A: Statistical Mechanics and its Applications 315(1-2), pp. 79-97, 2002.

20. K. Lee and W. Sung, "A stochastic model of conductance transitions in voltage-gated ion channels," Journal of Biological Physics 28(2), pp. 279-287, 2002.

21. S. H. Chung and S. Kuyucak, "Predicting channel function from channel structure using brownian dynamics simulations," Clinical and Experimental Pharmacology and Physiology 28(1-2), pp. 89-94, 2001.

22. G. Moy, B. Corry, S. Kuyucak, and S.-H. Chung, "Tests of continuum theories as models of ion channels. I. Poisson-Boltzmann theory versus Brownian dynamics," Biophys. J. 78(5), pp. 2349-2363, 2000.

23. J. Ferziger and M. Peric, Computational Method for Fluid Dynamics., Springer, Berlin, 1996.

24. P. Läuger, "Structural fluctuations and current noise of ionic channels," Biophys. J. 48, pp. 369-373, 1985.

25. A. B. Mamonov, R. D. Coalson, A. Nitzan, and M. G. Kurnikova, "The role of the dielectric barrier in narrow biological channels: A novel composite approach to modeling single-channel currents," Biophys. $J$. 84(6), pp. 3646-3661, 2003.

26. O. S. Andersen, R. E. Koeppe, and B. Roux, "Gramicidin channels," IEEE Transactions on Nanoscience 4(1), pp. 10-20, 2005.

27. J. A. Tuszynski and M. Kurzynski, Introduction to Molecular Biophysics, CRC Press, Florida, 2003.

28. D. G. Luchinsky, R. Tindjong, I. Kaufman, P. V. E. McClintock, and R. S. Eisenberg, "Ionic channels as electrostatic amplifiers of charge fluctuations," 2007. Electrostatics 2007 March 25-29, 2007' St Catherine's College, Oxford, UK.

29. B. Chanda, O. K. Asamoah, R. Blunck, B. Roux, and F. Bezanilla, "Gating charge displacement in voltagegated ion channels involves limited transmembrane movement," Nature 436(7052), pp. 852-856, 2005.

30. P. H. Barry and J. W. Lynch, "Ligand-gated channels," Ieee Transactions on Nanobioscience 4(1), pp. 70$80,2005$.

31. B. Martinac, "Mechanosensitive ion channels: molecules of mechanotransduction," Journal of Cell Science 117(12), pp. 2449-2460, 2004.

32. A. Auerbach, "Gating of acetylcholine receptor channels: Brownian motion across a broad transition state," Proceedings of the National Academy of Sciences of the United States of America 102(5), pp. 1408-1412, 2005. 